\title{
A kind of auto train braking performance test device
}

\author{
Jianhui $\mathrm{Di}^{1,}$, Jie Kang ${ }^{1, b}$ and Yan Zhao ${ }^{2, c}$ \\ ${ }^{1}$ Shijiazhuang HuaYan transportation science and technology limited company, \\ Shijiazhuang, 050081, China \\ ${ }^{2}$ Hebei Province People's Hospital, Shijiazhuang, 050057, China \\ a2511705376@qq.com, bkang.jie@126.com, '²385215931@qq.com
}

Keywords: auto-train; braking; detecting device

Abstract. A kind of test device used for auto-train braking performance is introduced. This test device can test automobile multiple axis (more than three axis) at the same time. Detect the braking sequence of each axis, the vehicle braking force, braking process and coordinate time. One braking process can realize the goal of detection of brake project (brake timing, the vehicle braking force, braking process and coordinated time)

\section{Introduction}

That cars can park brake in short-haul and stay in the process of moving in the direction of stability, is directly related to road traffic security problems. Especially in the automobile cargo transportation in China, all kinds of tractor trailer is a major freight powerhouse. And all kinds of freight car is multi-axle lorry, axis numbers reaches six axis, and the five or six axis cars accounted for most of the cars, the authenticity of the vehicle braking performance test directly affects the driving safety. Conventional brake inspection can only be measured axis of the poor, and its index, and can't detect the braking sequence of each axis, the vehicle dynamic braking force, braking process and coordinate time, but these indexes directly affects the motion of the vehicle safety. Therefore, we need to develop a detection device, providing a kind of multi-axle vehicle (more than three axis) of each wheel braking sequence detection, thus to multi-axle vehicle during braking, each wheel braking condition is analyzed, effective to avoid accidents.

\section{Development}

Operating principle. The adhering sand plate is adopted in mechanical part, and the long of sheet plate is not greater than the smallest length of the vehicles wheelbase (this guarantees each wheel in different braking test unit), on each side consists by 24 pieces of brake plate and a weighing board. Checked car is driven at a speed of about $10 \mathrm{~km} / \mathrm{h}$ on the tablets and fast braked. A sensor system is installed under the plate and the sensor changes the force signal into electrical signal, then the signal collection enlarges signal filtering and the signal into computer to be processed. Computer software organizes the data, and get the automobile braking performance data we need.

Mechanical structure. The overall effect of the device is as shown in figure 1, weighing unit is shown in figure 2 and brake testing unit in figure 3, including 2 weighing unit, 48 brake testing units, distribution in two rows. The third layer of chassis parts in before and after each row of adjacent two tablets is closely connected by bolts. The weighing unit is shown in figure 2, divided into first layer, second and third layer. The first layer is composed of the first pattern plate and through the screw fixed on the second weighing framework. The second layer is composed of weighing framework, which support the tire, and pass the force on the tire to underside 6 weighing sensor, weighing sensor 6 will changes the gathering force into electrical signal and transmits to a computer by amplified filter and AD conversion. Weighing sensor 6 is bolted on the third layer of weighing chassis 3 , but the weighing chassis is concreting on the ground. The limit bolt land 2 on weighing chassis 3 are keep the relative position between the weighing frame 2 and the weighing frame 3. Side cover 7 is fixed two sides through screws in weighing chassis to form protecting shell. 


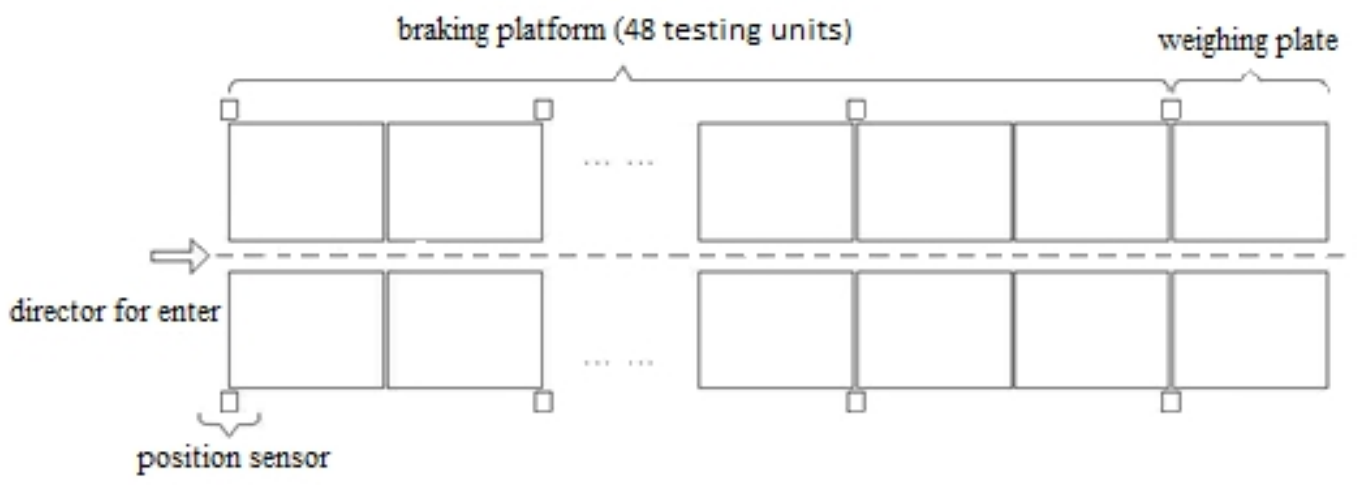

Fig. 1. Overall chart

Braking test units is shown in figure 3, and divided into the first, the second and the third layer, two pieces of adhering sand plate 1 and brake plate 2 shared a brake chassis 3 . The first layer is composed of adhering sand plate 1, the main function is to simulate the road surface the car driving on, and fixed through the screw on the second layer of the brake frame. The second layer is composed of braking framework, which plays the role of the support tire. Brake chassis frame 2 connects brake chassis 3 by brake sensor 5 and brake chassis 3 concreting on the ground. When the tires on the adhering sand plate 1 is in braking process, the braking sensor 5 will change the force into electrical signals and transmit to a computer by amplified signals filter and $\mathrm{AD}$ conversion. Roller 4 set between braking framework 2 and brake chassis 3 is to support the brake frame and to reduce the influence of the result of the brake. By adjusting the 6 bolts, limit block 7, limit block 8,and return spring 9 to keep the relative position between the brake frame 2 and brake chassis. Side cover 10 through screws in weighing chassis 3 sides to protect shell.

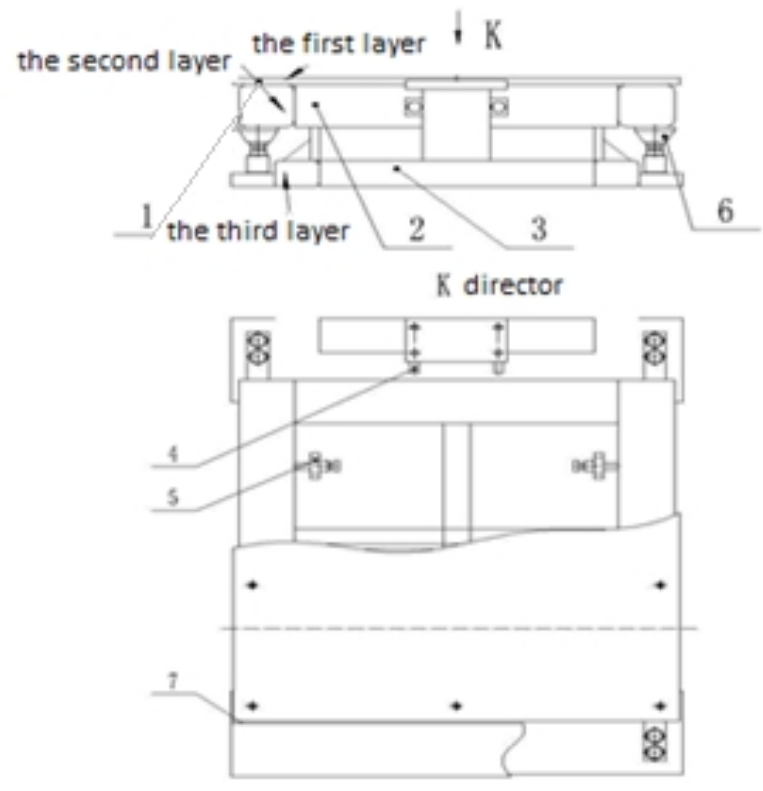

1.adhering sand plate 2 . weighing frame 3 . Weighing chassis 4 . Pedal stopper 1 5. Pedal stopper2 6. weighing sensor 7. Side plate

Fig. 2. Weighing unit

Electric System. Electrical systems is composed of control system, computer software. Control system is composed of industrial computer, $\mathrm{AD}$ adapter, IO adapter, signal amplification, conditioning circuit and infrared electric switch. This system will detect signal, gather the braking force signal and wheel weight signal. Computer software system adopts the popular Borland c++ Builder, using Microsoft SQL 2000 for data management system. That application connects to the 

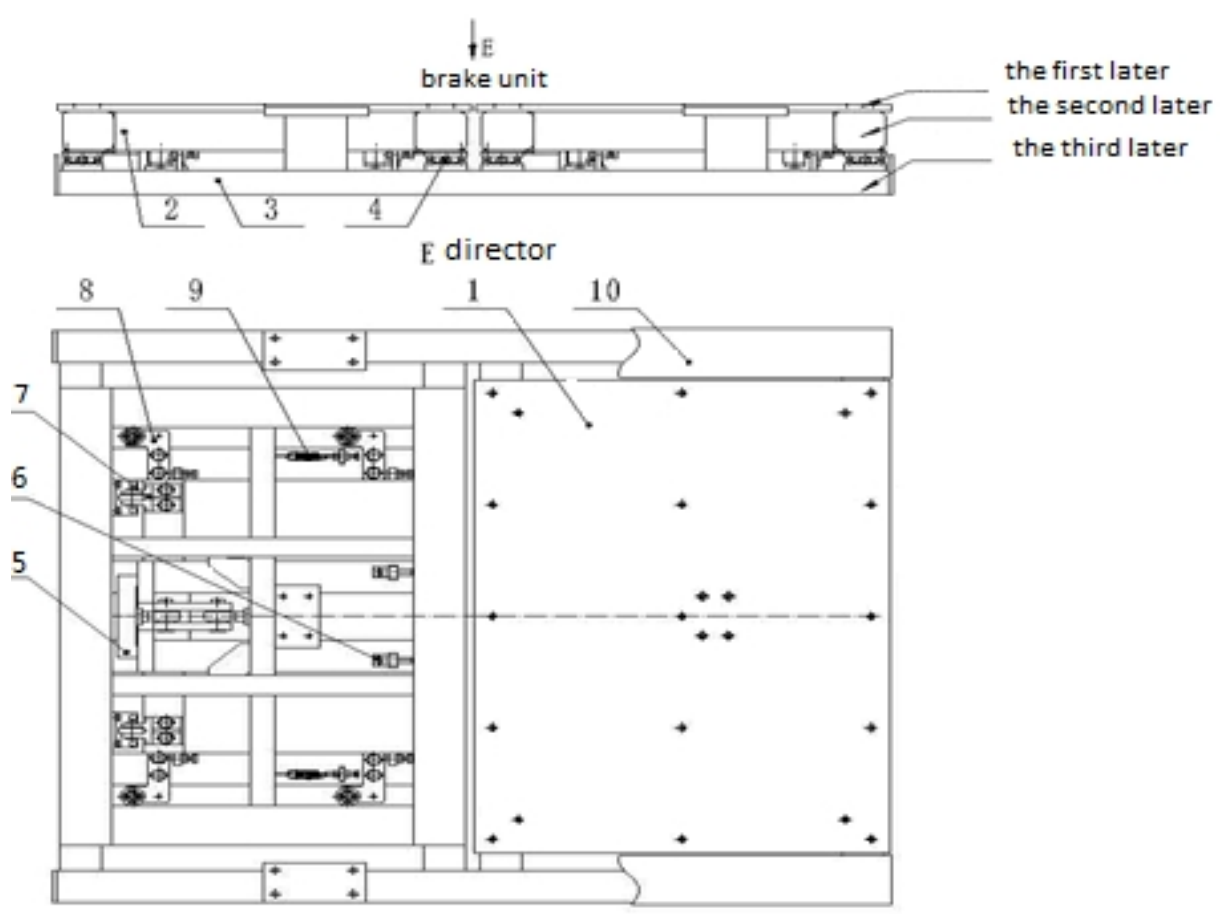

1.adhering sand plate 2. brake shaft bracket 3. Brake chassis 4. Roller 5. brake sensor 6. Pedal stopper 7. Limit catch wheel 1 8. Limit catch wheel 2 9. retruen spring 10. Side plate

Fig.3. Braking test unit

database ion provides a powerful guarantee to improve the efficiency of programming and the reliability of the data management. Detection software is responsible for scheduling, operation command, signal processing, and LAN exchange data, and other functions. The control system automatic completes peripherals action control, signal acquisition, data processing and printing work after the user completes login in vehicle information. Management software is equipped with inspection registration, fees, staff permissions distribution library, vehicle maintenance, test results query statistics. In sampling process, median filtering software first removes the noise points, then makes the braking force waveform Laplace transform and wavelet transform, then acquisition of braking stability is more accurate.

Detection process. After the vehicle information (number, vehicle type, license plate number, test category axis, etc.) log into the computer control system and enter into detection control mode, the system automatically enter a state of vehicle monitoring, the checked vehicle according to clew enters into $10 \mathrm{~km} / \mathrm{h}$ speed detection device, controlling system automatically tracks the process of the vehicle axle through the position sensor of by the cars of all the travel of the shaft in the plate and make sure each wheel brake on plate position, and monitor the data of current each wheel brake unit and a later braking testing unit. When the system monitor shows that the upper shaft number is in accord with the registration total number of shaft, it prompts the checked vehicle brakes and at the same time collect data for each testing unit of wheel brake and determine whether or not all data from the shaft into the continuous stable state. That the braking data keeping stability shows that the vehicle braking has been completed then the checked vehicle is driven out of the brake plate, passing the weighing plate and measured the weight for each wheel. At this point, completing the collection of vehicle monitoring for each shaft signal system, the control system analysis for signal and processing of shafts, brake index calculation, calculation of braking timing between axis. In the brake process the signal of each axle for right heavy, left heavy, left braking and right braking in the form of graphics render to control testing interface. 


\section{Conclusion}

After repeated comparison experiments confirmed that the system can detect the real brake timing of each axis, the vehicle braking force, braking process, coordination of time. Don't remove the trailer parts in the test, but directly make the vehicle detection. A braking process can detect crane brake project (brake timing, poor vehicle dynamic braking force, braking process, coordinate the time) and reduce the labor intensity of detection, improve the detection efficiency, increase the traffic safety factor. At the same time the system is suitable for different wheelbase cars and has realized the full coverage of all kinds of automobile brake performance test.

\section{References}

[1] GB 21861-2008 Items and methods of power-driven vehicles safety technology inspection.

[2] GB 7258-2012 Safety specifications for power-driven vehicle operating on roads.

[3] GB 18565-2012 Comprehensive performance requirements and detecting methods for road transport vehicle. 